\title{
Root Canals and Crowns: An Analysis of the Spatial Distribution of Dental Offices in Ciudad Juárez, Chihuahua, Mexico, 1996-2011
}

\author{
Patricia J. Boda ${ }^{1}$, James Harris ${ }^{2}$ \\ ${ }^{1}$ Department of Geosciences, Middle Tennessee State University, Murfreesboro, USA \\ ${ }^{2}$ Department of Earth and Atmospheric Sciences, Metropolitan State University, Denver, USA \\ Email: pboda@mtsu.edu,jharr115@msudenver.edu
}

Received June 9, 2013; revised July 11, 2013; accepted August 6, 2013

Copyright (c) 2013 Patricia J. Boda, James Harris. This is an open access article distributed under the Creative Commons Attribution License, which permits unrestricted use, distribution, and reproduction in any medium, provided the original work is properly cited.

\begin{abstract}
Every city above a certain size has health services that are located, to a large extent, close to their patients. This study analyzes the spatial distribution of dental office and the change in the number of dental offices in Ciudad Juárez, Chihuahua, Mexico over a 15-year time period. Research was conducted in 1996 and 2011. Maps showing the location of dental offices for these two years indicate a clustering of dental offices within one and one-half miles of the city's two principal border-crossing points between the United States and Mexico. Provider-based surveys were also used to determine the mix of patients (US or Mexican citizens) at the dental offices found throughout the city. By 2011, nearly all of the dental offices clustered within one and one-half miles of the two principal US-Mexico border crossings had 100\% of their patients from the United States.
\end{abstract}

Keywords: Cross-Border Health Care; Medical Geography; US-Mexico Border; Dental

\section{Introduction}

Utilization of particular health care providers has been studied for many years and has resulted in a myriad of research. Research relevant to this study includes the role that poverty and cultural preferences play in a person's decision to use a particular facility [1,2]. Other studies have determined that distance and travel time are key factors to utilization [3]. Most researchers agree that in order to use a facility, there must be access to it.

Access remains an elusive term however. In 1981, Penchansky and Thomas identified five dimensions of access as availability, accessibility, accommodation, affordability and acceptability. Availability delineates the supply of services in relationship to the demand for them. Accessibility defines the geographic barriers to receiving such services such as distance, transportation, and travel time to the facility. Accommodation refers to the degree that services meet the needs of patients and include hours of service, waiting times, appointments and scheduling. Affordability refers to the cost of services and acceptability describes how the provider interacts with the patient on a personal level. Acceptability includes potential barriers such as gender, ethnicity, language and sexual orientation [4]. Each dimension can interfere with true access by creating barriers that limit utilization of services.

The border region between the United States and Mexico is an area of varied cultures, diverse income levels, and dissimilar political environments. These differences create significant volumes of interaction between the two countries in the border towns. Americans routinely cross the US-Mexico border to obtain a variety of goods and services, including prescription drugs and dental work. Previous research regarding health care near the USMexico border has focused on the behavior of US patients who cross into Mexico for prescription medications [5-9], while others have examined the dangers and legal issues associated with doing so [10-14].

Crossing a border for dental care is not as well-documented as crossing for prescription medications. Much of the research on 'dental tourism' has been done in Europe and has focused on specific border crossings in Ireland, Belgium and other countries [15-17]. With few exceptions, crossing into Mexico from the US for dental care has not been well researched.

One group investigated the effect of the North American Free Trade Agreement (NAFTA) on accessibility to 
health care in 2002. Researchers believed that the free trade agreement might positively affect the "trade" of health care as well as other industries. However, they concluded that the "globalization effect" they expected due to NAFTA, had not promoted health policy cooperation between the US and Mexico. They found that those who crossed to Mexico for health care, including dental services, faced a variety of challenges including administrative, legal and cultural barriers [18].

Yet, in spite of those difficulties, researchers who conducted over 2500 telephone interviews during October and November of 2007 found that $32.5 \%$ of respondents from El Paso had crossed into Juárez for some type of health care [19]. Nearly $60 \%$ of those interviewed reported they had received dental care in Mexico. Byrd and Law found that those who reported Hispanic ethnicity, having ever lived in Juárez, or lacking health care were more likely to have crossed the border for medical and pharmaceutical services but not for dental care. Ethnicity, past residence and insurance coverage had no statistical relevance for accessing dental care in Juárez in this study.

This study investigates a seldom-mentioned or measured aspect of the issue - the spatial distribution of dental offices in Ciudad Juárez, Chihuahua, Mexico. A second component of this inquiry is to determine if the demand from US patients for dental work has expressed itself spatially on the urban landscape of Juárez from 1996 to 2011. This study is an extension of work conducted previously in El Paso, Texas and Ciudad Juárez, Chihuahua in 1996 and in 2007 [20,21].

Mapping and provider-based surveys were utilized to examine trends. They indicated that 1) the number of dental offices is growing at a rate faster than that of the population growth rate itself-suggesting that people living in the US visited Juárez, creating the demand for more dental offices and 2) the new dental offices in the study period have joined earlier facilities, clustering within one and one-half miles of the two main border crossings to better serve their primary customers, US residents.

There are three ports of entry into Ciudad Juárez: two in the north directly across from downtown El Paso, and one that enters Juárez from El Paso County from the east. "In 2011, more than 3.6 million passenger vehicles, 4.2 million pedestrians and 300,000 commercial vehicles crossed into Ciudad Juárez through the three bridges" [22]. Although there are three ports of entry, two principal border crossings between downtown El Paso and Ciudad Juárez account for the vast majority of crossings and are approximately 2 miles apart. The US Customs and Border Protection Agency recognized these crossing points as the two busiest border crossings in the world in 2011 [23]. Since these two crossings accounted for most of the traffic during the study period, they served as the focus of this research.

According to the Instituo Nacional de Estadistica y Geografica Informacion (INEGI), the Mexican equivalent of the US Census Bureau, Ciudad Juárez had a population of 995,770 in 1995 . By 2010, its population grew by $32.6 \%$ to $1,321,004$ [24]. The actual population of Juárez is difficult to determine because: 1) migrants flock to the city seeking employment in the maquiladoras (manufacturing operations in the free trade zone) and are often not counted in the census and 2) many residents leave the city due to violence $[25,26]$. Taking these issues into consideration, the population of Juárez in 2011 is thought to be approximately $1,298,004$. Using this number, the \% change from 1996 to 2011 is about 30.4\%. To keep pace with the increasing population, one would expect the number of dental offices in Juárez to increase in number at a similar rate during the study period.

\section{Data Sources and Research Methods}

The initial source used to locate dental offices was the Yellow Pages for El Paso and Ciudad Juárez in 1996 and 2011. In both the 1996 and 2011 studies, the same definition was used: Dental offices are facilities that advertised under the heading "Dentist."

Street files for Ciudad Juárez were purchased through INEGI. The file contained street and colonia (similar to census tracts) data, however street segments did not include any coordinate reference system. In addition, it did not contain address ranges, which prevented geocoding operations. Although address matching was not possible, the street and colonia files were imported into ArcMap for mapping purposes only. Streets, colonias and distances were accurate and were used to identify street names and define distances for manual placement of dental offices on the map.

A three-step process for manual placement of the offices onto the street map was performed. First, the street name of each facility was identified and located in the street file. A second query was performed to locate that section of street within a polygon, in this case, a colonia since this information was provided by the Yellow Page advertisement. The third step in the location process placed offices in a particular order on the street and within the colonia as follows:

*If an office were located on a short street, that is one to five blocks in length, then the site was located in the middle of the street section.

*If more than one office were located on any length street, then locations were divided evenly along that street.

As a result, offices were located on the correct street and in the correct colonia, however the exact location of each office on the street itself could be slightly inaccurate 
because each was manually placed in ArcMap. Since exact locations of many facilities were known, accuracy of the procedure was verified by overlaying "interpolated" locations with known locations. While some facilities resulted in less than a $100 \%$ match, most were within one block of exact locations and only one was located more than two blocks from its actual site. This process proved to be a useful method for locating features in cases where address matching is unavailable because it provided accurate placement of addresses along a street.

Once the dental offices were located and mapped, a cluster pattern was clearly visible. Based on the distribution of dental offices, Ciudad Juárez was divided into three zones based on distance from the two principal border crossing points: one and one-half miles (referred to as the border zone), one and one-half miles to three miles (referred to as the mid-zone), and greater than three miles (referred to as the outer zone).

Once the three zones were established, at least $20 \%$ of all the dental offices were selected in each zone for on-site interviews in 1996. The same questionnaire was used in 1996 and 2011 and, whenever possible, dentists interviewed in 1996 were questioned again in 2011 in order to make for valid comparisons. Interviews were conducted in a conversational style, employing Yin's methodology [27], which was designed to work more effectively people from different cultures.

\section{Findings}

\subsection{Number of Dental Offices}

Over the 15-year period from 1996 to 2011, the number of dental offices in Ciudad Juárez increased from 121 to 243 , an increase of $122 \%$ or $100.8 \%$ (Table 1). The number of dental offices within the border zone, within one and one-half miles of the two border crossing points, increased from 109 to 215. This represents an increase of 106 facilities, or $97.2 \%$. During the study period 122 new dental offices were opened, with $106 \%$ or $86.8 \%$ in the border zone.

As stated previously, the distance between the two main border crossing points is approximately two miles, and with the border zone of one and one-half miles from each crossing point, the border zone has an area of approximately 7.5 square miles. Most of the new facilities

Table 1. Number of dental offices in 1996 and 2011.

\begin{tabular}{ccc}
\hline Dental Offices & 1996 & 2011 \\
\hline Total Number & $121(48)^{*}$ & $243(68)^{*}$ \\
Border Zone & $109(28)^{*}$ & $215(45)^{*}$ \\
\hline
\end{tabular}

${ }^{*}$ Number of dental offices interviewed in (). opened in Juárez during the study period are in the border zone, which strongly suggests that these facilities serve US citizen demand for dental services.

\subsection{Distribution of Dental Offices}

The distribution of these facilities illustrates this further. Distribution of dental offices in Ciudad Juárez is distinct from the distribution in many other cities. In many cities dentists locate their offices near other health care facilities such as hospitals, clinics, and so forth resulting in what is referred to as an agglomeration of services. We also see neighborhood dental offices near commercial districts throughout the city. In 1996, dental offices in Juárez were clustered close to the two border crossings (Figure 1).

Although several dental offices in Juárez are found in other parts of the city, a clustering and concentration of dental offices is in the north-near the two US border crossing points. This was the case in 1996 and remains so in 2011. The map in Figure 2 demonstrates visually the preponderance of dental offices within one and one-half miles of the border crossings. As the inset maps show, in both years the facilities tended to be located near the border crossing points and along the main streets leading from those entry points.

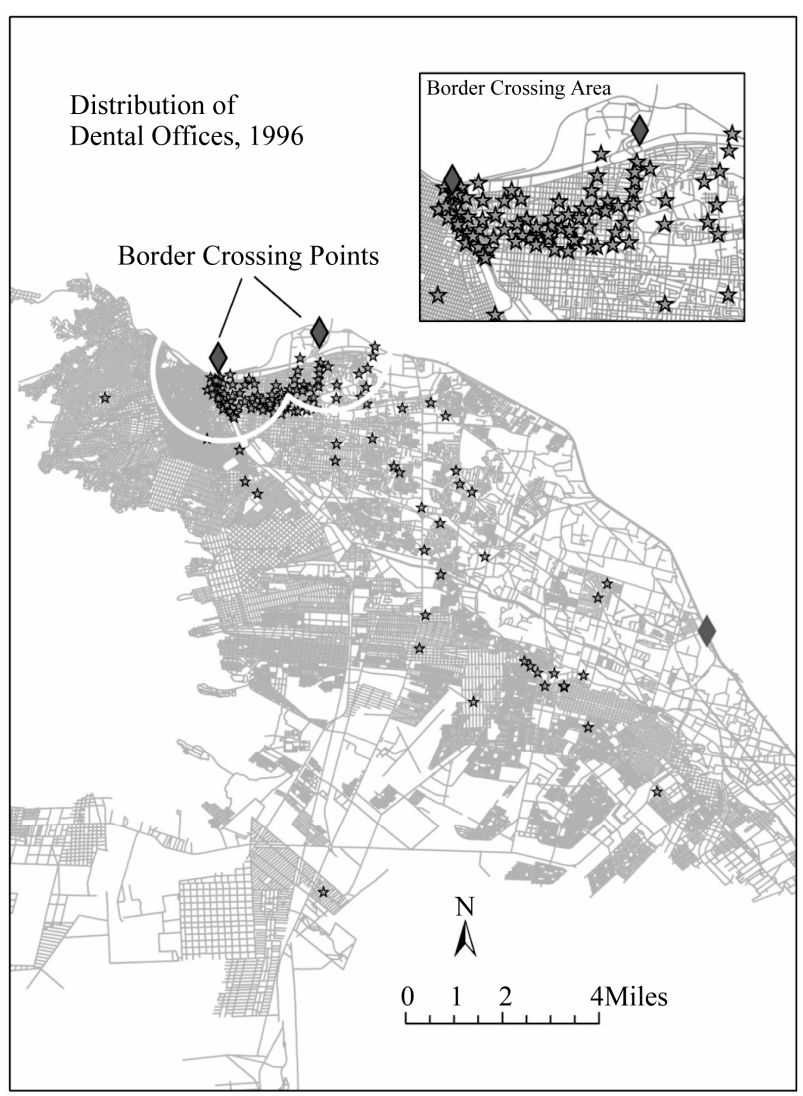

Figure 1. Distribution of dental offices, 1996. 


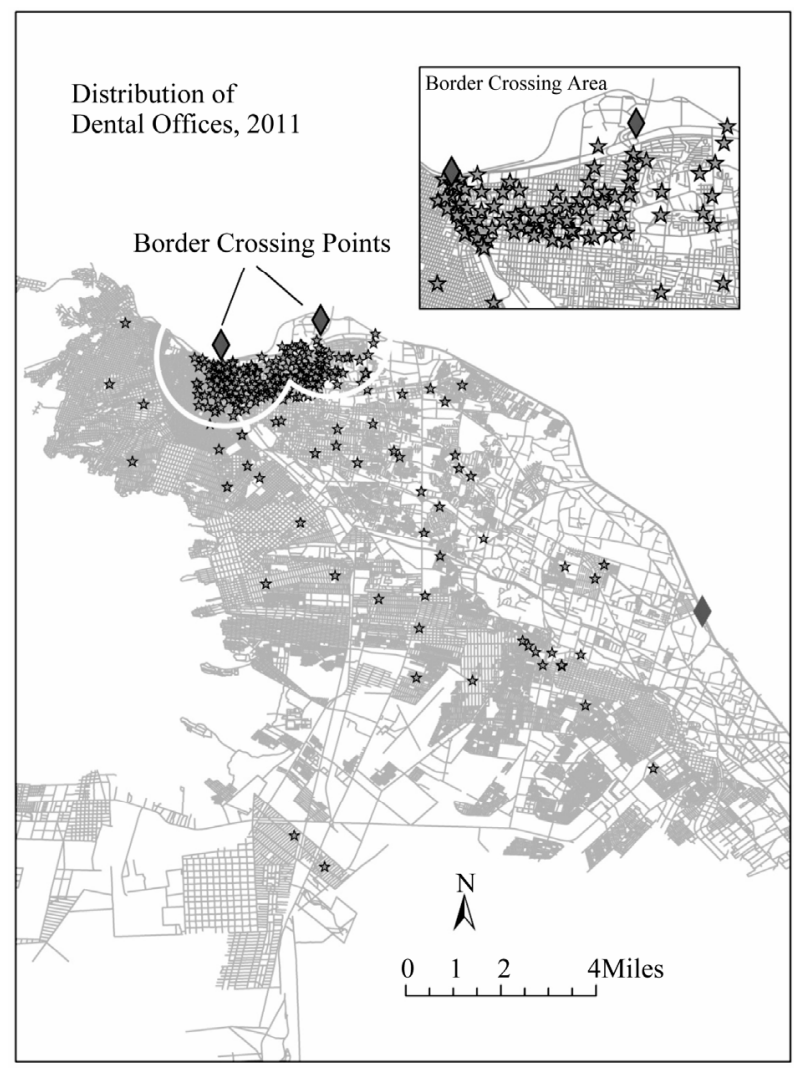

Figure 2. Distribution of dental offices, 2011.

\subsection{Dental Offices Primarily Serve US Residents}

Assuming that Juárez dentists locate their facility near their patients, as do most providers, the distribution of dental offices in Juárez indicates propinquity to US patients. Mexican dentists locate their facilities as a convenience to Americans making the trip from El Paso across the border into Juárez. The demand generated by US residents is measurable. Interviews with dentists in the border zone of Juárez indicate that most of their patients were from the United States (Table 2). In 2011, not all dental offices within one and one-half miles of the border crossings reported $100 \%$ use by US residents, however all dental offices that reported $100 \%$ of their clients were US residents were within the one and one-half miles of the border crossings. In 2011, a dentist just two blocks from the western border crossing point reported all of his patients were from the US, while in 1996, he reported 95\% were US residents. Two dentists within three blocks of the border crossing points also reported that all $(100 \%)$ of their patients were US residents in 2011, while in 1996 the same two reported only $80 \%$ of their patients were US residents.

Dental offices farther from the border consistently reported lower use by US residents. In the mid-zone, the dentists interviewed reported that patients who are US
Table 2. Percentage of total dental patients from US by zone.

\begin{tabular}{ccc}
\hline Dentists & 1996 & 2011 \\
\hline Border Zone & $50 \%-95 \%$ & $60 \%-100 \%$ \\
Mid-zone & $10-50$ & $20-35$ \\
Outer zone & $0-5$ & $1-10$ \\
\hline
\end{tabular}

residents dropped considerably, between $20 \%$ - 35\%. In 2011, no dental office more than one and one-half miles from the border crossing points reported more than $35 \%$ use by US residents. In other words, the farther from the border, the fewer patients were from the US (Figure 3).

\section{Summary and Future Research}

This study investigated the spatial distribution of dental offices in Ciudad Juárez, Mexico and attempted to determine if the demand from US patients for dental work had visually expressed itself on the urban landscape of Juárez from 1996 to 2011 in response to the US demand. Several interesting conclusion resulted from this study.

First, is that over the 15-year study period, the number of dental offices increased by $100.8 \%$. This might suggest that as the city grew so too did the number of dental offices; yet, the city grew by only $30.4 \%$ over the study period. US patients help to explain the increase in dental offices, especially when the neighborhoods closest to the border crossing points are examined. The number of dental offices in the border zone increased 97.2\%. Furthermore, $86.8 \%$ of new dental offices in the city were in the border crossing area. The data from the interviews and the number of dental offices clustered near the crossing points confirms that the primary customers for these services are US residents.

Second, while the number of facilities has increased considerably, the distribution has not changed. Dental offices continue to cluster within one and one-half miles of the two principal border-crossing points. As the maps and tables display, more than $83 \%$ of the dental offices are concentrated within one and one-half miles from the US border. This was true in 1996 and remains the so in 2011, due to the high volume of US residents who cross the border seeking dental services.

Third, interviews with dentists in all three of the zones confirm that Americans frequent dental offices closest to the two principal border crossings. Since the primary patients of dental offices near the border are US residents, it is reasonable to conclude that the concentration of these facilities close to the border were built to serve US residents traveling to Juárez for dental work.

Fourth, since there are more facilities near the border and these facilities reported a higher \%age of use by US patients, seems to suggest that there were more US 


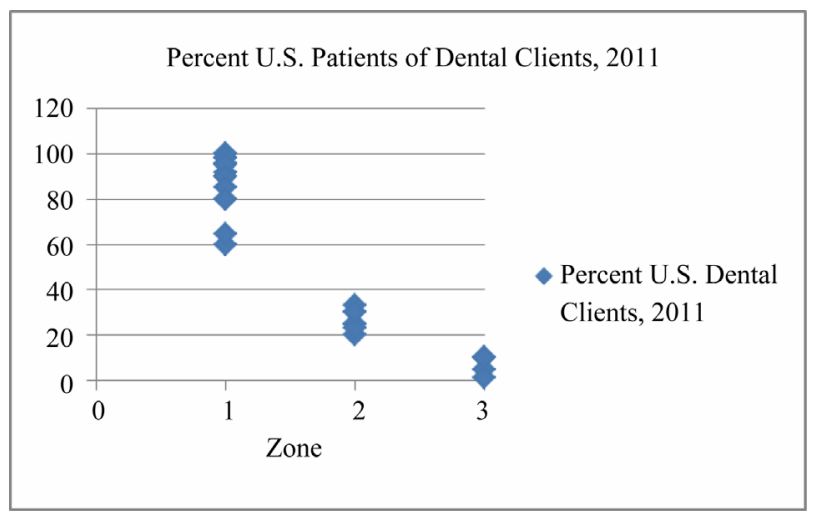

Figure 3. Use of dental offices by US residents by zone.

residents crossing into Juárez for dental services in 2011 than in 1996.

In summary, the city of Ciudad Juárez is still experiencing a great number of US residents who cross into Mexico for their dental care needs. The dentists in the city have responded to this continued, increasing demand by opening new offices near the border to serve this demand. The increase in the number of facilities is due to the US demand based on two pieces of evidence: the pattern of distribution and the growth in the number of border zone facilities that is disproportional to the growth of the city's population.

\subsection{Discussion}

In the case of Ciudad Juárez, it is clear that US residents accessed dental office in Ciudad Juárez during the study period. Placing this study within the context of the five dimensions of access described by Penchasky and Thomas [4], the primary reason for US residents to cross the border and enter a foreign country for dental care was affordability. The cost savings for dental services in Juárez are significant. Dental care in Juárez is often 50\% to $75 \%$ less than in the US, creating a compelling lure that draws many US residents. Since the savings are significant, barriers, such as proximity to home, become less important.

Affordability is followed closely by the accommodation of Mexican dentists by locating many offices close to the entry points from El Paso into Juárez, and operating their dental clinics for extended hours. In addition, cultural differences, which often can inhibit a patient from visiting a particular dentist, do not exist in Juárez. All dentists interviewed, regardless of their location spoke fluent English and were willing to file patients' insurance claims for them.

It is clear that many US residents are willing to tolerate the inconvenience of accessibility, in this case crossing an international border and spending additional time to travel a greater distance, in order to receive services at a low cost (affordability) that are offered by many providers (availability) who accommodate their schedules by offering extended office hours (accommodation), regardless of language, ethnicity or culture (acceptability).

\subsection{Further Research}

The high number of US residents who frequent dental offices in the border zone of Juárez raises several interesting questions for future research. First, what effect does this border crossing for dental services have on neighboring dental practices in US border cities, such as El Paso, Las Cruces, Yeleta and other cities along the border? And what is the areal extent for these services? Presumably there would be higher numbers of Juárez dental patients living in El Paso, but how far into the US does the lure of inexpensive dental care extend? Do patients fly into the El Paso Airport, making the trek across the border for dental services? Address matching, or geocoding, the addresses of patients would determine the actual reach the Juárez dentists have into the US.

A second area of interest is the background and characteristics of those who cross into Mexico for health care and the need to address the cultural nature of the USMexico border to identify which populations are most likely to cross borders for health care. Is it a case, as Su and Wang documented, where second and third generation Mexican-Americans are less likely to cross than the newcomers [28], or are they the elderly who are priced out of US health care as Families USA Foundation pointed out [6]? Clearly, since cost is the primary motivating factor for the dental tourism taking place in Juárez, the uninsured would likely be the largest group to cross for these services. However, since many US insurers are now paying for dental services performed in foreign countries, the number of insured may be more significant that previously researched.

An additional area of concern is the quality of the dental services received outside the US, where standards and educational requirements often vary. A study on rates of crown, implants and filling failure would disclose if the cost savings for the Juárez services is indeed worth the final expense. Are there higher rates of infection that result in extractions and additional costs involved with patients who receive dental care outside their own country?

The clustering of dental offices by the border crossing suggests that perhaps similar concentrations of other medical services demanded by Americans may exist. For example, pharmacies, where Americans often purchase prescription drugs, and hospitals, where Americans go to have procedures done, everything from cosmetic surgery to oncology treatments may have similar spatial signatures as dental offices. 
This study documents that the number of US residents who travel to Juárez for dental care is substantial and the number has risen during the past fifteen years. It also suggests that similar patterns of use are possible in other areas of health care as well. If there are negative consequences to such a practice, the financial cost ultimately could be significant.

\section{REFERENCES}

[1] C. Brach and L. Fraser, "Can Cultural Competency Reduce Racial and Ethnic Disparities? A Review and Conceptual Model,” Medical Care Research and Review, Vol. 57, Suppl. 1, 2000, pp. 181-217.

[2] M. Welch, "Required Curriculum in Diversity and CrossCultural Medicine: The Time Is Now," Journal of the American Medical Women's Association, Vol. 53, Suppl. 3, 1998, pp. 121-123.

[3] J. Fortney, N. Chambers, M. Cody and C. Beck, "Geographic Access and Service Use in a Community-Based Sample of Cognitively Impaired Elders,” Journal of Applied Gerontology, Vol. 21, No. 3, 2002, pp. 351-367. doi:10.1177/073346480202100305

[4] R. Penchansky and J. W. Thomas, "The Concept of Access: Definition and Relationships to Consumer Satisfaction,” Medical Care, Vol. 19, No. 2, 1981, pp. 127-140. doi:10.1097/00005650-198102000-00001

[5] P. R. Casner and L. G. Guerra, "Purchasing Prescription Medication in Mexico without a Prescription. The Experience at the Border," Western Journal of Medicine, Vol. 156, No. 5, 1992, pp. 512-516.

[6] “Going to Mexico: Priced out of American Health Care," Families USA Foundation, Washington DC, 1992, p. 3.

[7] E. P. Macias and L. S. Morales, "Crossing the Border for Health Care," Journal of Health Care for the Poor and Underserved, Vol. 12, No. 1, 2001, pp. 77-87. doi:10.1353/hpu.2010.0643

[8] M. Landeck and C. Garza, "Utilization of Physician Health Care Services in Mexico by U.S. Hispanic Border Residents,” Health Marketing Quarterly, Vol. 20, No. 1, 2002, pp. 3-16. doi:10.1300/J026v20n01_02

[9] L. G. Escobedo and V. M. Cardenas, "Utilization and Purchase of Medical Care Services in Mexico by Residents of the United States of America, 1998-1999," Pan American Journal of Public Health, Vol. 19, No. 5, 2006, pp. 300-305. doi:10.1590/S1020-49892006000500002

[10] "Purchasing Prescription Drugs from Foreign Countries and Reducing Drug Costs," California State Board of Pharmacy, Pamphlet, 2003.

[11] “Attitudes about Importing Medication,” National Association of Chain Drug Stores, Pamphlet, NACDS Foundation, Public Opinion Research, Alexandria, 2003.

[12] J. A. Pagan, S. Ross, J. Yau and D. Polsky, "Self-Medication and Health Insurance Coverage in Mexico,” Health
Policy, Vol. 75, No. 2, 2005, pp. 170-177. doi:10.1016/j.healthpol.2005.03.007

[13] "Consumer Information: Buying Medications from Outside the United States," US Food and Drug Administration, Pamphlet, Center for Drug Evaluation and Research, Silver Spring, Maryland, 2006.

[14] US Customs and Border Protection Agency, "Buying Prescription Medicine from Internet Foreign Pharmacies," 2010.

[15] J. Jamison, M. Butler, P. Clark, M. McKee and C. O’Neill, "Cross-Border Co-Operation in Health Services in Ireland,” A Report for Centre for Cross Border Studies, Dublin, 2001.

[16] L. Turner, “Cross-Border Dental Care: 'Dental Tourism' and Patient Mobility,” British Dental Journal, Vol. 204, 2008, pp. 553-554. doi:10.1038/sj.bdj.2008.403

[17] A. Österle, P. Balazs and J. Delgado, “Traveling for Teeth: Characteristics and Perspectives of Dental Care Tourism in Hungary,” British Dental Journal, Vol. 206, 2009, pp. 425-428. doi:10.1038/sj.bdj.2009.308

[18] N. Homedes and A. Ugalde, "Globalization and Health at the U.S.-Mexico Border," American Journal of Public Health, Vol. 93, No. 12, 2003, pp. 2016-2022. doi:10.2105/AJPH.93.12.2016

[19] T. Byrd and J. Law, “Cross-Border Utilization of Health Care Services by United States Residents Living near the Mexican Border,” Revista Panamericana de Salud Publica, Vol. 26, No. 2, 2009, pp. 95-100. doi:10.1590/S1020-49892009000800001

[20] P. Schwarz, "International Health Care Refugees of the U.S.-Mexico Border,” M.A. Thesis, University of Minnesota, Minneapolis, 1996.

[21] P. Boda, "International Trade of Health Care along the U.S.-Mexico Border: The Case of El Paso and Ciudad Juárez,” Ph.D. dissertation. University of Minnesota, Minneapolis, 2007.

[22] City of El Paso, 2013. http://home.elpasotexas.gov/bridges/

[23] US Customs and Border Protection Agency, "U.S. Border Patrol Fiscal Year 2011 Profile Report,” Department of Homeland Security, 2011.

[24] “Catalogue of Population, Housing and Household Data," Instituo Nacional de Estadistica y Geografica Informacion, 2010.

[25] R. Carroll, “Mexico Drug War: The New Killing Fields,” The Guardian, 3 September 2010.

[26] D. Borunda, "Two U.S. Citizens Killed as Gunmen Attack SUV in Juárez,” El Paso Times, 3 October 2011.

[27] R. Yin, “Interviews as Conversations,” In: R. Burgess, Ed., In the Field, Harper Collins, New York, 1984, pp. 83-99.

[28] D. Su and D. Wang, "Acculturation and Cross-Border Utilization of Health Services,” Journal of Immigrant and Minority Health, Vol. 4, No. 4, 2011, pp. 563-569. 